\title{
Oxidative Stress Index as A Public Health Survey Instrument
}

\author{
Harold I. Zeliger
}

\begin{abstract}
Recently, a method for assigning the probability of disease onset to all people, those clinically ill as well as those without prevalent disease, has been described and termed the Oxidative Stress Index (OSI). The OSI has also been used to show which disease causing parameters are more contributory to the onset of Alzheimer's disease and projected a similar capability for other diseases. A third application of the OSI is presented here. It can be used as a public health survey tool to shed light on the health impacts on people living or working proximate to points of pollutant releases.
\end{abstract}

\section{INTRODUCTION}

People with chronic diseases and those who are disease free but living unhealthy lifestyles are known to be candidates for numerous non-communicative diseases, as well as more frequent and more severe bouts with infectious diseases [Zeliger 2016]. Recently, a method for assigning the probability of disease onset to all people, those clinically ill as well as those without prevalent disease, has been described and termed the Oxidative Stress Index (OSI). This method is a non-invasive diagnostic protocol based upon a questionnaire addressing oxidative stress (OS) elevating factors that include genetics, disease status, lifestyle and environmental exposure with the sum of the positive answers equal to the OSI [Zeliger, 2017, 2019]. It has been recently shown that the OSI is an indicator of parameters that elevate the likelihood of the onset of Alzheimer's disease and projected be so used to demonstrate which parameters may be associated with the onsets of other diseases [Zeliger 2019a].

The OSI, as originally formatted, does not predict which disease will more likely develop with increasing OS, only that further disease is predicted with increased OS, as numerically shown by elevated OSI values. It is proposed here that the OSI may also be used to carry out public health surveys to determine increased risks for disease onset to individuals who are chronically exposed to environmental pollutants such as air pollutants emanating from chemical and petroleum storage and transfer sites, power plant and other industrial stacks, leaking toxic landfills, heavy metal recycling plants and other OS elevating factors such as electromagnetic radiation. Using the OSI, members of communities that are impacted by chronic environmental spills and releases can be surveyed to determined how proximity to release points and durations of exposure can predict the likelihood of disease onset.

Published on June 17, 2019.

H. I. Zeliger is with the Zeliger Research \& Consulting, 41 Wildwood Drive, Cape Elizabeth, ME 04107, USA. (e-mail: hiz@ zeliger.com)

\section{METHODS}

The hypothesis proposed here is based upon a literature review of published studies on the causes of OS and OS induced disease, methods of measuring OS and the empirical and mechanistic associations between OS and disease onset [Zeliger 2016 and the numerous references contained therein. The questionnaire used to determine OSI contains questions that address all OS raising factors, including genetic factors, environmental exposures, lifestyle and current disease status and treatment.

\section{RESULTS AND DISCUSSION}

\section{A. Oxidative Stress}

It is well known that all disease is accompanied by elevated OS, a property whose status can be measures by a number of biomarkers, including F2-Isoprostanes, lipid hydroxides and hydroperoxides, hydroxycholesterols, aldehydes and ketones [Zeliger, 2016]. Of these, the most widely used is malondialdehyde (MDA), which is stable in serum, as well as readily and accurately analyzed for [Nielsen, et al, 1997]. MDA level, as well as other biomarkers, however, can vary widely depending upon food eaten, environmental exposures, state of one's health or even the time of day when serum is drawn [Nielsen, et al, 1997]. The OSI questionnaire reduces such uncertainty by addressing multiple aspects of a person's regular life, including disease status, medications, diet, employment, environmental exposures, and stress, etc., that elevate OS [Zeliger 2016].

\section{B. Dose Response Relationship}

Total Oxidative Stress has been shown to be related to disease onset, whether coming from single sources or from combinations of multiple sources, in a dose response relationship [Zeliger, 2016]. Hence, the OSI can reliably predict the likelihood of disease onset in people exposed to OS elevating sources when compared to cohorts not so exposed [Zeliger 2017, 2019a].

\section{Late Onset of Disease}

OS caused diseases manifest themselves only after long term (years or decades) exposures to causative agents. Examples of such diseases are cardiovascular diseases, type 2 diabetes, respiratory diseases (including asthma and COPD), numerous cancers and neurological diseases (including Alzheimer's disease). Genetic factors as well as chronic exposures to OS raising chemical pollutants and radiation are well known to be associated with these late 
onset diseases [Swaroop, et al., 2009; Zeliger and Lupinski, 2015].

\section{Questionnaires}

Questionnaires, such as the Charlson Comorbidity Index, are routinely used to solicit patient background information on prevalent diseases, symptoms and likelihood of further disease onset [Charlson et al., 1987]. The Alzheimer's Questionnaire is another example [Sabbagh, et al., 2010].

\section{E. The Oxidative Stress Index In Health Surveys}

It is well known that chronic exposures to chemical pollutants are a cause of numerous environmental diseases. For example, living proximate to heavily traveled highways results in a greater likelihood of respiratory disease onset and that the likelihood declines in a linear fashion as place of residence is distanced from such highways, where airborne concentrations of vehicle exhausts also decline linearly [Huynh, et al, 2010]. Accordingly, the closer one resides to a heavily traveled highway, the greater the exposure to exhaust pollutants and the greater the oxidative stress impact on one's body. Thus, using the OSI, it becomes possible to numerically assign probabilities of disease onset related to such exposure as a function of distance from a disease causing emission source. Adding distancing questions to the OSI and surveying individuals at varying distances from a pollutant emission source up to distances where these emission provide zero impact, i.e., where levels of the pollutant equal ambient background levels up to background levels enables the one to assess the pollution (and hence the oxidative stress) impact of the emission. It also enables the establishment of safe residential distances from the particular emission source. The OSI questionnaire has been modified to accomplish just this. The modified version of the OSI is shown in Table 1.

\section{DISCUSSION}

As all factors known to raise OS have been incorporated into the modified OSI questionnaire shown in table 1, it now becomes possible to determine a person's total OS status that incorporates chronic exposure to a pollutant source. The OSI is non-invasive, yet predicts OS levels and offers insights into which parameters are the most contributory to disease onset. Though all individuals with disease symptoms will require medical diagnosis and treatment, using the OSI does not require invasive testing to determine how a community is being impacted by a chronic chemical or radiological exposure when statistically significant numbers of people are thus evaluated. Individual items in the OSI are presented in alphabetical order and may differ widely from one item to the next. This is deliberate so that an individual or surrogate answering the OSI questions is required to consider each item alone, rather than a part of a series of related questions, which could cause the responder to just check all the items in a single set. When used as a public health survey instrument, the OSI is able to factor out disease causes other than a particular well defined exposure by incorporating control cohorts into the survey. Thus the disease impact of a particular causative agent can be determined.

\section{LIMITATIONS}

The public health survey version of the OSI asks respondents to estimate distances from emission sources to their residences to their residences and to report prevalent diseases. With a small number of individual respondents, these can lead to errors. With statistically significant numbers of respondents, however, such limitations are eliminated and reliable survey results regarding the health impacts of toxic environmental contaminant exposure can be determined.

\section{CONCLUSIONS}

The hypothesis presented here is that the modified version of the Oxidative Stress Index presented here is potentially a valuable non-invasive tool that can be used to carry out public health surveys to assess the health impacts of chronic chemical and radiological emissions on members of impacted communities.

\section{REFERENCES}

Charlson ME, Pompei P, Ales KL. (1987). A new method of classifying prognostic comorbidity in longitudinal studies: development and validation. J Chronic Dis; 40(5):373-383.

Huynh P, Salam MY, Morphew T, Kwong KYC, Scott L. Residential proximity to freeways is associated with uncontrolled asthma in innercity Hispanic children and adolescents. J Allergy (2010). Volume 2010 Article ID 157249, 7 pages. doi:10.1155/2010/157249. Accessed June 6, 2019.

Nielsen F, Mikkelsen BB, Niesen JB, Andersen HR, Grandjean P. (1997). Plasma malondialdehyde as biomarker for oxidative stress: reference interval and efforts of life-style factors. Clin Chem 43(7):1209-1214.

Sabbagh MN, Malek-Ahmadi R, Belden CM, Connor DJ, Pearson C, Jacobson R, et al. (2010). Alzheimers Dis 22(3):1015-1021.

Swaroop A, Chew EY, Rickman CB, Abecasis GR. (2009). Unraveling multifactorial late-onset disease: from genetic susceptibility to disease mechanisms for age-related macular degeneration. Annu Rev Genomics Hum Genet 10:19-43. doi:10.1146/annrev.genom.9.081307.164350. Accessed June 6, 2019.

Zeliger HI. (2016). Predicting disease onset in clinically healthy people. Interdiscip Toxicol 9(2):15-21.

Zeliger HI. (2017). Oxidative stress index predicts disease onset. J Med Res Prac 6(3):86-92.

Zeliger HI. (2019). A Pound of Prevention for a Healthier Life. Universal Publishers, Irvine, CA and Boca Raton, FL.

Zeliger HI. (2019a). Predicting Alzheimer's disease onset. European Journal of Medical and Health Sciences. 1(1). http//doi.org/10.24018/ejmed.2019.1.1.16.

Zeliger HI, Lipinski B. (2015). Physiochemical basis of human degenerative disease. Interdiscip Toxicol 8(1):39-54.

\section{APPENDIX}

TABLE 1. OXIDATIVE STRESS INDEX AS A PUBLIC HEALTH SURVEY INSTRUMENT

OXIDATIVE STRESS INDEX (OSI) CHECK LIST

DISTANCE OF HOME FROM CLOSEST POLLUTION EMISSION

SOURCE (PETROLEUM OR CHEMICAL STORAGE TANK,

LANDFILL OR OTHER CHEMICAL OR RADIATION EMITTING

SOURCE). Check only one

0.1 mile or less 0.6 mile $\quad 0.7$ mile 1.5 miles

0.2 mile $\quad 0.7$ mile $\quad 1.6-2.0$ miles

0.3 mile $\quad 0.8$ mile $\quad 2.0-2.5$ miles

0.4 mile $\quad 0.9$ mile $\quad 2.5-3.0$ miles

0.5 mile $\quad 1.0$ mile $\quad 3.0$ miles or more

Years at current residence (enter number of years) 


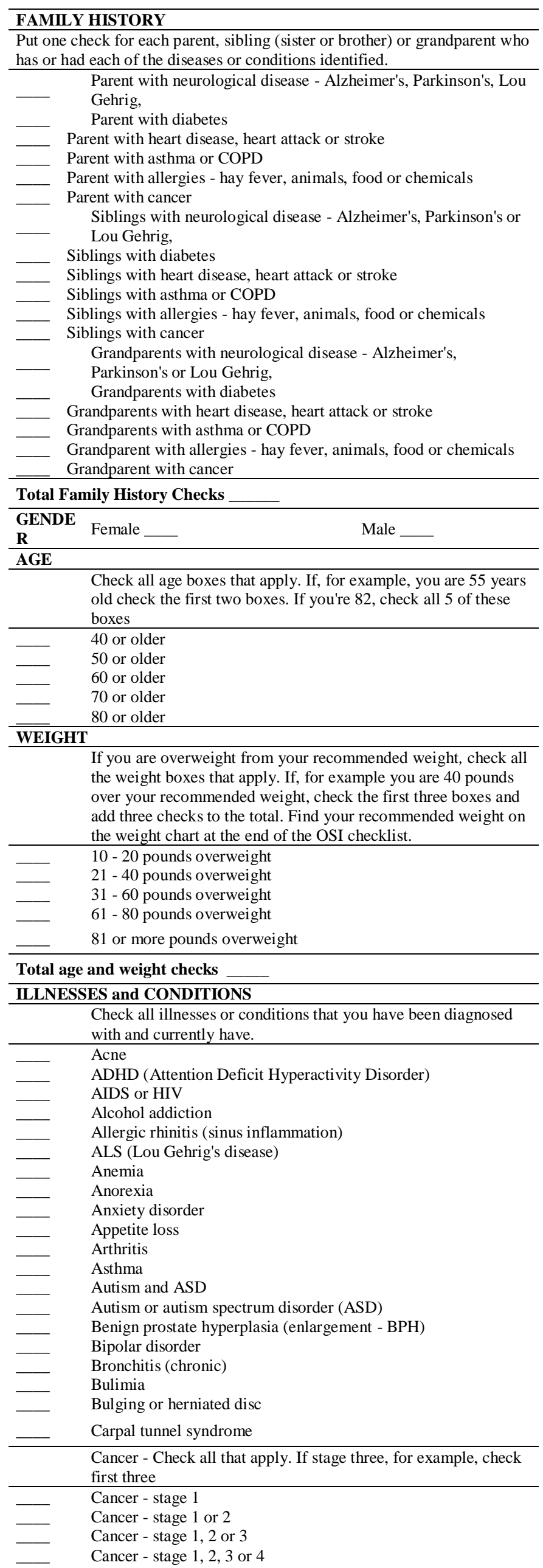

Cardiovascular disease

Chronic Fatigue Syndrome (CFS)

Crohn's disease

Common cold (frequent)

COPD (chronic obstructive pulmonary disease)

Crohn's disease

Dementia

Dengue fever

Dental abscess (frequent)

Depression

Diabetes (type 1 or type 2)

Diarrhea (frequent)

Diverticulitis

Drug addiction

Eczema

Emphysema

Endometriosis

Epilepsy

Fibromyalgia (FM)

Flu

Frequent common colds

Frequent headaches

Frequent indigestion

Frequent infection

Frequent itching

Frequent rashes

Frequent sinus infections (sinusitis)

Glaucoma

Gout

Gum disease

Heart attack

Heart Disease or heart problems

Hemorrhoids

Hepatitis B (chronic)

High blood pressure (hypertension)

High cholesterol

Herpes

Inflammatory bowl disease

Irritable bowel syndrome (IBS)

Kidney disease

Leukemia

Liver cirrhosis

Liver disease

Lupus

Lyme disease

Macular degeneration

Malaria

Metabolic syndrome (pre-diabetes)

Middle ear infection (frequent)

Migraine headaches

Multiple chemical sensitivity (MCS)

Multiple sclerosis

Obesity

Osteoarthritis

Osteoporosis

Parkinson's disease

Periodontal disease (swollen or bleeding gums)

Post-traumatic stress disorder (PTSD)

Rocky Mountain spotted fever

Psoriasis

Rosacea

Schizophrenia

Seizures

Sexually transmitted disease (STD)

Shingles

Sjogren's syndrome

Sleep apnea

Stroke

TB (Tuberculosis)

Thyroid disease

Tourette syndrome

Tremors

Ulcers

Varicose veins

West Nile Fever

Yellow fever

Zika

Write in the names of any other illnesses you have that were not listed above and check those. 


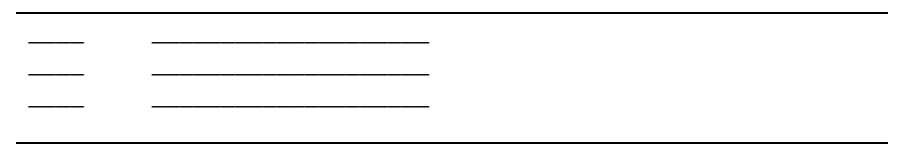

Total Disease Checks

\begin{tabular}{l} 
DISEASE START \\
\hline How many of the diseases checked above started in \\
\hline$\quad$ The past 2 years? \\
The past 5 years? \\
The past 10 years? \\
\hline SYMPTOMS \\
\hline Check all the symptoms that you currently experience.
\end{tabular}

Abdominal pain (frequent)

Allergic reactions to chemicals

Allergic reactions to any foods

Allergic reactions to insects

Allergic reactions to medications

Allergic reactions to plants (Hay fever)

Ankle pain

Attention span decline

Anxiety often

Bleeding gums

Blood in stool

Blood in urine

Blurred or cloudy vision

Bruise easily

Burning when urinating

Butterflies in your stomach often

Change in skin color

Chest pain

Constant chills

Constipation

Cough that is persistent

Coughing or spitting up blood

Decision making difficulties

Decline in learning ability

Decreased eye sight

Decreased sex drive

Diarrhea (frequent)

Difficulty completing familiar tasks

Difficulty concentrating

Difficulty getting warm

Difficulty maintaining balance

Difficulty solving problems

Difficulty swallowing

Difficulty walking

Difficulty concentrating or finding words

Dizziness

Drained of energy

Dreams that are bizarre and recurring

Excessive mucous production

Excessive thirst

Eye discomfort or pain

Eye redness

Fatigue

Feel depressed a lot

Feel less alert or fuzzy headed

Fever

Food allergies

Foot pain

Foot swelling

Fungal infection such as athlete's food that persists

Frequent urination

Graying of hair

Hair loss (not due to chemotherapy)

Have itchy scaly skin rashes

Headaches frequently

Hear voices inside you

Hearing loss that comes on suddenly

Heart palpitations (throbbing)

Heartburn

Hip pain

Hoarseness

Increased susceptibility to infections

Indigestion (frequent)

Insomnia

Irregular periods

Itchy hands

Itchy skin other than hands
Jaw pain

Leg swelling

Learning new things more difficult

Light headedness

Long recovery time from infections

Losing track of time

Loss of coordination

Loss of muscle tone

Loss of taste

Lower back pain

Memory loss

Mood swings from very high to very low and vice versa

Mouth sores that don't go away quickly

Muscle aches that last a long time

Muscle cramps

Muscle spasms

Nasal congestion

Nausea

Neck pain

Nervousness

Nightmares regularly

Nose bleeds

Knee pain

Numbness or tingling in hands or feet

Pain in joints

Heart palpitations

Pelvic pain

Perspire (sweat) profusely

Post nasal drip that lingers

Post traumatic stress disorder (PTSD)

Problems finding the words you want

Rapid hair loss

Rapid heartbeat

Scaly skin

Seizures

Shortness of breath

Shoulder pain that lingers

Sinus pain

Skin mole growth

Skin rashes

Sleep less than 7 hours per night

Sleep more than 9 hours a night

Slow to heal from cuts, bruises or other injuries

Slurred speech

Smaller field of vision

Sore throat that doesn't heal

Stressed out most or all of the time

Stuffy nose

Swollen eye lids

Tics (involuntary movements)

Tingling in the hands or feet

Tire easily

Tired most of the time

Tooth pain

Tremors

Twitching

Unusual vaginal bleeding or discharge

Urination difficulty

Urination pain

Varicose veins

Vomiting

Wake up more than 3 times per night

Weakness

Weight gain

Weight loss (rapid)

Wheezing

Wrinkling or loss of tone in skin

Yawning frequently

Total Symptoms Checks

\section{TEST RESULTS}

The following address results obtained from test doctors ordered done as part of annual examinations. Check all that apply to you.

High or low blood sugar

High or low BUN (blood urea nitrogen)

High or low calcium

High or low carbon dioxide (bicarbonate)

High or low chloride

High cholesterol

High or low creatinine 
High glucose

High or low potassium

High PSA

High or low sodium

High triglycerides

Low blood oxygen

Low potassium

\section{Total Test Results Checks}

\section{PRESCRIPTION MEDICINES REGULARLY TAKEN}

Check each of the boxes that apply. If you regularly take five prescription drugs, for example, check all of the first 5 items, so that the total number of items checked equals the total number of prescriptions regularly taken.

1 prescription

2 prescriptions

3 prescriptions

4 prescriptions

5 prescriptions

6 prescriptions

7 prescriptions

8 prescriptions

9 prescriptions

10 or more prescriptions

Have a heart pacemaker

\begin{tabular}{l}
\hline Total Prescription Medicine Checks \\
\hline DIET
\end{tabular}

Check each item that applies to the foods that are part of your regular eat.

Alcoholic beverages (beer, wine, spirits) more than 1 drink per day

Artificial sweeteners for coffee or tea

Canned or frozen cooked foods regularly eaten (soups, pastas, meats)

Bread and pasta made primarily from white processed flour

Fast food frequently eaten

Fewer than 3 fruits or vegetables a day

Grilled, smoked or blackened meat, chicken or fish

Food high in fat (whole milk, cheeses, foods cooked with butter and animal fat)

Often eat processed foods (bacon, hot dogs, salami, sausages.

deli meats

Eat red meat more than 2 times a week

Eat foods high in sugar (sweetened drinks and desserts) Salty food

\section{Total Diet Checks}

\section{LIFE STYLE}

These items refer to where you live, the type of work you do and chemicals you may be exposed to

Are a farmer that regularly uses pesticides

Burn wood for heat or for cooking

Constantly use a cell phone

Drink chlorinated water

Drink more than one alcoholic drink per day

Exercise less than one half hour a week
Have mold in your home

Have new (less than 6 months old) carpet in your home

Have pets in your home that you are allergic to

Live down wind from a smoking industrial chimney

Live in a city with air quality alerts

Live or work close to a cell tower

Live or work near high voltage electrical transmission lines

Live near a heavily traveled highway or road

Live near a landfill

Live with a smoker

Regularly experience allergic reactions in your home

Regularly experience allergic reactions in your work place

Regularly use room or furniture deodorants

Regularly play contact sports

Tobacco use. Check all of the items that apply. For example, if you smoke one pack of cigarettes a day, check both of the first 2 items. If you smoke two packs a day, check all of the first 4 items.

Smoke 10 cigarettes or less daily (even just one)

Smoke a pack a day (20 cigarettes)

Smoke a pack and a half a day (30 cigarettes)

Smoke two packs a day (40 cigarettes)

Smoke more than two packs a day

Smoke 1-5 cigars a day

Smoke 6 or more cigars a day

Use smokeless tobacco

Work as a toll booth collector

Work in very hot or very cold conditions regularly

Work in an adhesives or coatings manufacturing plant

Work in an agricultural chemical manufacturing plant

Work as an automobile, diesel or aircraft mechanic

Work with chemicals on the job regularly

Work in a dusty environment regularly

Work in a landfill

Work in a hair or nail salon

Work in metal refinery or mill

Work as a miner

Work in a noisy environment

Work in a paint, lacquer, stain or varnish manufacturing plant

Work as a painter

Work as a pilot or flight attendant

Work as a pesticide applicator

Work in a petroleum refinery

Work in a plastics manufacturing plant

Work in a plywood or particle board manufacturing plant

Work in a polluted environment (road paver, toll booth operator,

for example)

Work in a water or sewage treatment plant

Work in wood treatment plant

Total Lifestyle Checks

OSI, Total of All Checks 